only $2 \%$ of men with a PSAV $<0.4 \mathrm{ng} / \mathrm{ml} /$ year were diagnosed as having prostate cancer compared with $13 \%$ of those with a PSAV above this threshold $(P<0.0001)$. On multivariate analysis, incorporating age, ethnicity and family history of prostate cancer, PSAV $>0.4 \mathrm{ng} / \mathrm{ml} /$ year was the most significant predictor of prostate cancer (odds ratio 6.2, 95\% Cl 5.1-7.5; $P<0.0001)$.

The authors conclude that a PSAV threshold of $0.4 \mathrm{ng} / \mathrm{ml} /$ year should be used to indicate a need for prostate biopsy in men with serum PSA levels $<4 \mathrm{ng} / \mathrm{ml}$.

Original article Loeb S et al. (2007) Prostate specific antigen velocity in men with total prostate specific antigen less than $4 \mathrm{ng} / \mathrm{ml}$. J Urol 178: 2348-2353

\section{ADT for prostate cancer could increase the risk of death from cardiovascular causes}

Combination use of androgen deprivation therapy (ADT) and local therapy for localized prostate cancer is increasing; however, patients treated with ADT have an increased risk of developing the metabolic syndrome and, subsequently, cardiovascular disease. Tsai and colleagues conducted a retrospective study to investigate whether patients with localized prostate cancer treated with ADT also have an increased risk of death from cardiovascular events.

The study analyzed data from patients who had undergone prostatectomy $(n=3,262)$ or had been treated with external beam radiotherapy, brachytherapy or cryotherapy $(n=1,630)$ for localized prostate cancer. ADT had been received by 266 patients who underwent prostatectomy and 749 patients treated with the other therapies; median duration of ADT use overall was 4.1 months. During follow-up (median 3.8 years), 131 patients died of cardiovascular causes.

After controlling for age and cardiovascular disease risk factors, among patients who underwent prostatectomy, both use of ADT and older age were associated with an increased risk of death due to cardiovascular events. Furthermore, 5-year cumulative incidence of cardiovascular deaths in patients aged $\geq 65$ years who had undergone prostatectomy was $5.5 \%$ among patients who had received ADT but only $2.0 \%$ among those who had not.
ADT use also increased 5-year cumulative incidence of deaths from cardiovascular events in patients $\geq 65$ years who had received radiotherapy, brachytherapy or cryotherapy, but the results were not significant.

Prospective studies are necessary to confirm these findings; nevertheless, patients considered for ADT should undergo a careful cardiovascular evaluation.

Original article Tsai HK et al. (2007) Androgen deprivation therapy for localized prostate cancer and the risk of cardiovascular mortality. J Natl Cancer Inst 99: 1516-1524

\section{Novel lateral-cutting resectoscope: a method for safer TUR?}

Understaging and incomplete tumor removal are common problems with transurethral resection (TUR) of bladder tumors, and TUR is also associated with complications such as bladder perforation and sphincter damage. Pantuck et al. have evaluated a novel resectoscope, in which the linear movement of the handgrip is converted into a bidirectional lateral rotating motion of the cutting element. Different sizes of cutting loop are available, depending on the procedure to be performed.

The study included 80 patients, 38 with bladder cancer and 42 with benign prostatic hyperplasia. No safety concerns were evident, and the surgeons adapted easily to the new instrument. No bladder perforations were reported, and some surgeons noted reduced bleeding compared with standard TUR. For bladder tumor resection, the lateral movement permitted accurate depth of penetration into the bladder wall. The ability to control resection depth might reduce the risk of perforation and improve assessment of depth of invasion. For prostate resection, dissection of the adenoma adjacent to the verumontanum and prostatovesical junction was facilitated by the new element, possibly reducing the risk of injury to the urinary sphincter and bladder neck.

The authors conclude that the lateral-cutting resectoscope might lead to improved outcomes in TUR procedures, permitting more accurate bladder resection and fewer complications than existing instruments.

Original article Pantuck AJ et al. (2007) A novel

resectoscope for transurethral resection of bladder tumors and the prostate. J Urol 178:2331-2336 